Markus Nussbaumer

\title{
Maschinelle Übersetzung
}

Eine Revolution (nicht nur) für die Sprachdienste der Bundesverwaltung?

Beitragsart: Mitteilungen 
[1] Die Sprachdienste der Bundesverwaltung erbringen die Sprachdienstleistungen nach den Artikeln 6 und 10 der Sprachdiensteverordnung (SpDV, SR 172.081). Es sind dies in erster Linie Übersetzungsleistungen; insbesondere die zentralen Sprachdienste der Bundeskanzlei erbringen jedoch teilweise oder sogar überwiegend andere Leistungen, namentlich die Überprüfung amtlicher Texte (Erlasse, Botschaften, Berichte) auf ihre Verständlichkeit und die Übereinstimmung der drei Sprachfassungen im Rahmen der Verwaltungsinternen Redaktionskommission (VIRK). ${ }^{1}$ Die Sprachdienste sind über die Departemente und Ämter der ganzen Bundesverwaltung verteilt, also dezentral organisiert; in der Bundeskanzlei gibt es zudem die zentralen Sprachdienste, die gewisse Koordinationsfunktionen für die dezentralen Sprachdienste wahrnehmen. In den Sprachdiensten der Bundesverwaltung arbeiten circa 420 Sprachfachleute (zumeist Übersetzer/innen) auf circa 330 Vollzeitstellen. Von den ungefähr 36000 Vollzeitstellen der Bundesverwaltung sind also etwas weniger als 1 Prozent Sprachdienststellen.

[2] Die Arbeit des Übersetzens hat sich in der Bundesverwaltung wie überall sonst in den letzten 20 Jahren sehr verändert: Das Übersetzen ist einfacher, produktiver, effizienter geworden, dank den Recherchemöglichkeiten im Internet, der Online-Zugänglichkeit sehr vieler Texte, der Möglichkeit der Suche in elektronisch zur Verfügung stehenden Texten, den schnellen Austauschmöglichkeiten zwischen Auftraggeber und Übersetzerinnen und Übersetzern und zwischen Letzteren (per Mail, durch Desktopsharing u. a.). Dies machte es möglich, dass mit einem nur unbedeutend angewachsenen Stellenbestand heute sehr viel mehr Texte in kürzerer Zeit und vermutlich auch in besserer Qualität übersetzt werden können als noch vor 20 oder 30 Jahren.

[3] Nebst diesen neuen Möglichkeiten und Erleichterungen im Arbeitsalltag - die eigentlich gar nicht übersetzungsspezifisch sind, sondern die Verwaltungstätigkeit generell prägen - kamen in den letzten 20 Jahren mit Computerprogrammen zur Unterstützung des Übersetzens - sogenannten CAT-Tools (computer aided oder computer assisted translation) - spezifische Hilfsmittel für das Übersetzen hinzu. Zu diesen Tools kann man auch Terminologiedatenbanken zählen, die insbesondere in fachsprachlichen Bereichen feste Ausdrucksentsprechungen zwischen Sprachen sowie Begriffsdefinitionen anbieten. Die schweizerische Bundesverwaltung hat ihre eigene Terminologiedatenbank TERMDAT. Unter einem CAT-Tool versteht man jedoch in erster Linie ein Computerprogramm mit einem Übersetzungsspeicher, einem translation memory, in dem möglichst viele, sich entsprechende Textsegmente (Wörter, Wortgruppen, Teilsätze, Sätze) in zwei oder mehr Sprachen gespeichert sind, weil sie bereits einmal übersetzt worden sind. Übersetzt man einen Text, so kann man einzelne Textsegmente des Ausgangstextes in ein Suchfeld eingeben - oder, wenn das Tool in das Textverarbeitungssystem integriert ist, einfach markieren - und das Tool nach mehr oder weniger übereinstimmenden Segmenten in der Zielsprache suchen lassen, und je grösser, reichhaltiger und möglicherweise spezifisch auf bestimmte Domänen eingeschränkt ein translation memory ist, desto eher bietet das Programm bereits früher übersetzte Segmente an, die mehr oder minder genau mit dem zu übersetzenden Textsegment übereinstimmen. Damit wird man im Übersetzen schneller, und man vermeidet es auch, bereits früher Übersetztes immer wieder neu und anders zu übersetzen. Wichtig ist festzuhalten, dass mit einem CAT-Tool immer noch in erster Linie der Mensch übersetzt; das Tool hilft ihm dabei, indem es ihm zu einzelnen Segmenten des Ausgangstextes Übersetzungsvorschläge macht.

1 Weitere Informationen zu den Aufgaben und der Organisation der Sprachdienste finden sich unter www.bk.admin.ch > Unterstützung der Regierung > Sprachen. 
[4] Anders ist das bei Computerprogrammen des maschinellen Übersetzens (machine translation): Das sind Computerprogramme, in die man einen ganzen Ausgangstext eingibt, und die Maschine übersetzt den gesamten Text innert Sekunden. Bis vor wenigen Jahren war der Output solcher Übersetzungsmaschinen von schlechter Qualität und bot regelmässig Stoff für Glossen, Lachnummern - so «schräg» waren diese Übersetzungen sehr oft, in aller Regel kaum bis gar nicht zu gebrauchen und für die Übersetzerinnen und Übersetzer eher hinderlich als nützlich. Das hat sich jedoch seit etwa 2016 radikal verändert dank dem Einsatz von künstlicher Intelligenz, neuronalen Netzwerken, in solchen Programmen des maschinellen Übersetzens, im Unterschied zu älteren Programmen, die stärker regelbasiert oder aufgrund statistischer Berechnungen operieren. Diese neueren Programme «lernen», werden mit dem Translation Memory laufend «trainiert». Furore gemacht hat etwa das Programm DeepL (für «Deep Learning»), das Übersetzungsresultate von erstaunlicher Qualität liefern kann. Gewisse Versionen von DeepL sind mittlerweile im Internet gratis verfügbar, und immer mehr Menschen nutzen dieses Werkzeug.

[5] Dies ist auch so in der Bundesverwaltung. Die Bundesverwaltung ist eine mehrsprachige Verwaltung. Als Mitarbeiterin oder Mitarbeiter des Bundesverwaltung ist man täglich mit Texten in anderen Sprachen konfrontiert, die man verstehen möchte, und man produziert selber Texte, von denen man möchte, dass sie auch in andern Sprachen einer bestimmen Leserschaft zur Verfügung stehen. Die Sprachdiensteverordnung sagt eigentlich klipp und klar, dass Sprachdienstleistungen - und damit das Übersetzen - «in der Regel von den Sprachdiensten der Bundesverwaltung erbracht werden» (Art. 10 Abs. 1 SpDV). Wer Bedarf an einer Übersetzung hat, muss an die Sprachdienste gelangen, also einen bestimmten Geschäftsprozess wählen. Das braucht Zeit, manchmal muss man sich dabei auch «outen» als jemand, der nicht über die nötigen passiven Kompetenzen in den andern Amtssprachen verfügt und deshalb auf Übersetzung angewiesen ist, und manchmal bekommt man als Auftraggeber von den Sprachdiensten auch zur Antwort, dass es sich um einen rein internen Text handle, der nicht übersetzt werde, weil von den Mitarbeiterinnen und Mitarbeitern der Bundesverwaltung erwartet werden dürfe, dass sie Texte in den anderen Amtssprachen verstehen (Art. 20 Abs. 1 Sprachengesetz, SpG, SR 441.1; Art. 8 Sprachenverordnung, SpV, SR 441.11; Ziff. 3.6 sowie Anhang der Sprachweisungen der Bundeskanzlei, BBl 2017 3577). Wenn man dann DeepL zur Hand hat und damit die Aussicht, innert Sekunden und völlig kostenlos eine einigermassen brauchbare Übersetzung zu bekommen, ist die Versuchung sehr gross, diesen Weg einzuschlagen. Tatsächlich kann man seit Kurzem beobachten, dass in der Bundesverwaltung immer öfter DeepL eingesetzt wird. Übrigens auch in den auf das Übersetzen spezialisierten Sprachdiensten der Bundesverwaltung selber.

[6] Wie sollen sich die Sprachdienste da verhalten? Eine erste Reaktion ist verständlicherweise ein Gefühl von Bedrohung: Kommen da Maschinen, die mich früher oder später ersetzen werden? Und eine naheliegende Reaktion darauf wäre: Das muss man verbieten! Und anstatt sich auf die Sache mit kritischer Neugier einzulassen, argumentiert man grundsätzlich: «Niemals wird die Maschine Texte verstehen können, und niemals wird sie deshalb übersetzen und mich als Übersetzer/in ersetzen können!» - Die Bundeskanzlei mit ihrer Koordinationsfunktion für die Sprachdienste der Bundesverwaltung hat sich für einen anderen Weg entschieden, aus dem Wissen heraus, dass sich technologische Entwicklungen nicht aufhalten lassen, dass man sie aber genau beobachten, kritisch begleiten, ihre Risiken und ihre Chancen ausloten und zu einem übergeordneten Nutzen in das eigene Arbeits- und Aufgabenfeld integrieren sollte, wo es sinnvoll ist. Die Bundeskanzlei hat entschieden, die Bundesverwaltung mit dieser Entwicklung nicht einfach alleine zu lassen, sondern ihre Führungsrolle wahrzunehmen und den Umgang mit diesen neuen 
Möglichkeiten in geordnete Bahnen zu lenken. Sie hat darum 2019 circa 100 Lizenzen für DeepL gekauft und in erster Linie unter den Sprachdiensten der Bundesverwaltung selber verteilt und diese das neue Instrument ausprobieren lassen. Von den Probandinnen und Probanden hat sie eine detaillierte Berichterstattung über die Erfahrungen verlangt.

[7] Aus diesen Erfahrungen ist ein internes Merkblatt zuhanden all derer in der Bundesverwaltung entwickelt worden, die DeepL oder ein anderes Programm der maschinellen Übersetzung online benutzen möchten. Das Merkblatt hält fest:

(1) Solche Programme dürfen nicht benutzt werden für die Übersetzung von klassifizierten Texten (intern, vertraulich oder geheim) und von Texten, die sonst wie sensible Informationen oder Personendaten jeglicher Art enthalten. Der Grund hierfür ist, dass der Text die eigenen Server verlässt, wenn er zur Übersetzung hochgeladen wird, und dass mit ihm Text-Thesauri (translation memories) gefüttert werden, die auch andern Nutzerinnen und Nutzern zur Verfügung stehen. Das ist insbesondere bei der im Internet frei verfügbaren Version von DeepL der Fall; DeepL-Pro-Versionen, für welche die Bundeskanzlei Lizenzen gekauft hat, sollen das angeblich verhindern, doch so ganz sicher weiss man das nicht.

(2) Maschinelle Übersetzungen sind nur für den «Eigengebrauch» zulässig: wenn man also vor einem fremdsprachigen Text steht und ungefähr wissen möchte, was sein Inhalt ist. Aber selbst da wird zu grosser Skepsis geraten, denn die Übersetzungen können zwar durchaus sprachlich gut, das heisst grammatikalisch praktisch fehlerfrei daherkommen, das heisst sie «klingen gut» und wirken ganz unverdächtig. Inhaltlich aber können sie dennoch völlig «danebenliegen».

(3) Verboten ist die Verwendung solcher Tools für die Übersetzung von Texten, die veröffentlicht oder in Arbeitsprozessen durch andere Personen weiterverwendet werden sollen; solche Texte sind durch die Sprachdienste der Bundesverwaltung zu übersetzen (ob diese dabei ebenfalls die Maschine verwenden, den Output der Maschine jedoch überprüfen, ist ihnen überlassen).

[8] DeepL muss - und kann vermutlich auch - noch besser werden. So übersetzt es noch immer Satz für Satz, ohne Rücksicht auf satzübergreifende Kontexte. Das schwächt die Ergebnisse der Übersetzungen teilweise deutlich. DeepL ist in gewissen Übersetzungsrichtungen deutlich schwächer als in andern; so beispielsweise deutlich schwächer bei IT > DE und klar stärker bei FR $>$ DE. DeepL in der Hand ausgebildeter Übersetzerinnen und Übersetzer, die die Schwächen sofort erkennen und korrigieren können, kann ein sehr nützliches Hilfsmittel sein.

[9] CAT-Tools können gerade für die Übersetzung juristischer Normtexte interessant sein, weil diese stark musterorientiert sind, das heisst nach dem Grundsatz «Gleiches gleich und Ungleiches ungleich» oftmals (in Teilen) aus vorformulierten Mustersätzen bestehen, und weil in juristischen Normtexten die satzübergreifenden Bezüge vermutlich geringer sind, juristische Normtexte stärker als andere Texte aus für sich stehenden Sätzen bestehen. Eine maschinelle Übersetzung ist hier möglicherweise eher schwächer, weil das Programm sich nicht an den Grundsatz «Gleiches gleich und Ungleiches ungleich» hält. Gerade bei juristischen Normtexten ist in jedem Fall eine sehr penible Prüfung der maschinellen Outputs und darüber hinaus sicher auch eine «händische» Übersetzung weiterhin höchstes Gebot. 
[10] In der Bundesverwaltung läuft der Versuchsbetrieb mit DeepL und mit dem genannten Merkblatt weiter. Die Bundeskanzlei prüft zurzeit, wie es im Bereich des maschinellen Übersetzens in der Bundesverwaltung weitergehen könnte. Soll über eine öffentliche Ausschreibung ein Tool «ab Stange» angeschafft werden, oder ist eine Eigenentwicklung, zusammen mit darauf spezialisierten Partnern, ins Auge zu fassen? Und wer soll dereinst mit so einem Tool arbeiten dürfen? Für die eingehende Prüfung dieser Fragen und den weiteren Weg, wenn Entscheidungen einmal gefallen sind, hat die Bundeskanzlei ein Kompetenzzentrum Sprachtechnologie eingerichtet. Es soll sich im Sinne der Koordinationsfunktion der Bundeskanzlei im Sprachdienstebereich um die Themen CAT-Tool, maschinelle Übersetzung und Terminologiearbeit kümmern. Das CAT-Tool wurde in der Bundesverwaltung vor Kurzem zum Standarddienst erklärt; das bedeutet, dass es in der ganzen Bundesverwaltung nur noch ein CAT-Tool gibt, während bisher mehrere parallel betrieben wurden. Dieses einheitliche CAT-Tool wurde mittlerweile mit einem Ausschreibungsverfahren nach den WTO-Regeln beschafft und wird zurzeit in den Sprachdiensten eingeführt.

[11] So viel kann gesagt werden: Der Beruf des Übersetzers und der Übersetzerin wird nicht aussterben. Aber er wird sich stark verändern. Instrumente wie DeepL können das menschliche Übersetzen in Bereichen verdrängen, in denen schnelles und lediglich ungefähres Erfassen des Inhalts ausreicht und von Falschübersetzungen keine grössere Gefahr ausgeht. CAT-Tools und Übersetzungsmaschinen können das Arbeitstempo des Übersetzens steigern, sie können eine Menge an kreativen Inputs im Übersetzungsprozess liefern und in Teilen auch die Qualität verbessern. Die ausgebildeten Übersetzerinnen und Übersetzer werden jedoch stärker zu Kontrolleuren des Outputs der Maschine, wie das in sehr vielen andern Arbeitsbereichen teilweise schon seit Längerem der Fall ist. Das heisst: Das Übersetzen verschiebt sich vom kreativen Schaffen eines Zieltextes aus dem Verständnis des Ausgangstextes heraus immer mehr zu einem PostEditing, zu einem Kontrollieren, Überarbeiten und Korrigieren sowie Veredeln dessen, was die Maschine geleistet hat. In einem Tätigkeitsgebiet, in dem es um Verstehen von Kommunikation und damit um etwas Urmenschliches geht, mag das mehr schmerzen als in andern Bereichen menschlicher Verrichtungen.

[12] Besser als maschinelles Übersetzen wäre auf jeden Fall individuelle passive Mehrsprachigkeit, das heisst wären Mitarbeiterinnen und Mitarbeiter in der mehrsprachigen Institution Bundesverwaltung und wären Bürgerinnen und Bürger in diesem Land, die keine Übersetzungen brauchen, weil sie mehrere Sprachen beherrschen. Das würde die Menschen reicher machen, ist aber aufwendiger und wohl in Teilen eine Illusion. Wenn Übersetzungsmaschinen sich auf Kosten dieser individuellen Kompetenzen breitmachen würden, wäre das schade. Aber es folgte einer gewissen ökonomischen Logik.

Markus Nussbaumer, Bundeskanzlei. 